\title{
Una interpretazione geometrica del gruppo totale di sostituzioni sopra sei elementi.
}

\author{
(Di Edgardo Ciani, a Genova.)
}

Una permutazione sopra $n$ elementi può sempre considerarsi come una collineazione fra $\mathrm{i}$ punti di uno spazio lineare a $n-2$ dimensioni. Basta a tale scopo osservare, anzi tutto, che una tale collineazione è individuata dando $n$ coppie di punti corrispondenti: dopo di che si riguardino gli $n$ elementi come $n$ punti generici dello spazio in parola, eppoi riferendo ciascun elemento (e quindi ciascun punto) a quello che lo sostituisce per opera della permutazione suddetta, si osservi che verranno a individuarsi $n$ coppie di punti corrispondenti i quali serviranno a stabilire conseguentemente la cercata collineazione. Ne segue che qualsiasi gruppo di sostituzioni sopra $n$ elementi si può rappresentare mediante un gruppo di collineazioni fra $n$ punti di uno spazio lineare a $n-2$ dimensioni (il quale gruppo possiede come figura invariante quella costituita dall'insieme degli $n$ punti rappresentanti degli elementi dati). Per conseguenza anche il gruppo totale sopra gli $n$ elementi dati trova così una naturale interpretazione geometrica, nel gruppo totale di tutte le collineazioni che permutano in tutti i modi possibili $n$ punti generici dello spazio in parola.

Il presente scritto è un modesto saggio di questa interpretazione geometrica nel caso particolare di $n=6$ e quindi potrebbe anche intitolarsi come uno studio della configurazione dell'esagono nello spazio lineare a 4 dimensioni.

Sono ben note altre e certamente più importanti (se non ugualmente semplici) interpretazioni geometriche di gruppi di sostituzioni sopra sei elementi. Citerò, ad esempio, una Memoria assai elaborata di Gerbaldi che è inerente al gruppo alterno $\left(^{*}\right)$. Ma più particolarmente citerò un ben conosciuto lavoro di Veronese sull'argomento perchè ha qualche punto di con-

(*) GERBaLd, Sul gruppo semplice di 360 collineazioni piane. Questi Rendiconti, T. XII, pp. 23-94; T. XIII, pp. 161-199; T. XIV, pp. 66-114; T. XVI, pp. 199-154. 
tatto col mio, pure essendone diverso il concetto fondamentale $(*)$. L'lllustre autore assume (cf. il $n .^{\circ} 8$ di quel lavoro) un punto di coordinate $y_{1} y_{2} \ldots y_{n}$ in uno spazio $R_{n-1}$ a $n-1$ dimensioni e considera gli $n$ ! punti che si ottengono permutando in tutti i modi possibili gl'indici delle coordinate suddette. Alla permutazione che cambia la successione $1,2,3, \ldots, n$ nella successione $k, l, m, \ldots, p$ egli fa corrispondere la collineazione espressa dalle formule:

$$
p x_{1}^{\prime}=x_{k} ; p x_{2}^{\prime}=x_{l} ; \rho x_{a}^{\prime}=x_{m} ; \ldots \rho x_{n}^{\prime}=x_{p}
$$

e quindi egli trasforma il gruppo totale sopra $n$ elementi nel gruppo totale di collineazioni sopra un certo numero di punti costituiti dagli $n$ ! suddetti a cui si aggiunge necessariamente il punto unità (manifestamente invariante per tutte le collineazioni descritte). È così che l'A. interpreta, in particolare, il gruppo totale sopra sei lettere, nello spazio a cinque dimensioni, assumendo come gruppo invariante quello dei 720 punti che si ottengono permutando in tutti i modi possibili le coordinate di un punto generico in tale spazio e aggiungendovi il punto unità. Io invece interpreto il gruppo totale di sostituzioni su sei elementi, nello spazio a 4 dimensioni, mediante il gruppo totale di 720 collineazioni che permutano in tutti i modi possibili sei punti generici di tale spazio. La rappresentazione geometrica del Veronese è dunque diversa dalla mia.

Siccome la cfz." che io considero è quella dell'esagono in $S_{\sharp}$, così credo opportuno premettere le seguenti osservazioni d'indole generica sopra le cfz. ${ }^{\text {ni }}$ che hanno per nucleo di origine un poligono di $n$ vertici. Intanto sarà elemento essenziale lo spazio $S_{r}$ di dimensione minima contenente il dato poligono: per cui $n>r$. Ma per $n=r+1$ i vertici del poligono sono tutti indispensabili per individuare lo spazio ambiente. $S_{r}$. In tal caso lo studio della cfz." dal punto di vista proiettivo è infecondo perchè mancano i così detti spazi diagonali della cfz.." Così è infecondo lo studio proiettivo délla coppia di punti su di una retta, del triangolo nel piano $\left({ }^{* *}\right)$, del tetraedro

(*) VERONeSE, Interprétations géométriques de la théorie des substitutions de $n$ lettres, particulièrement pour $n=3,4,5,6$ en relation avec les groupes de l'Hexagramme mystique (Ann. di Mat., s, II, T. XI, pp. 93-236).

(**) Non è inutile forse osservare che la geometria del triangolo è essenzialmente geometria metrica e quindi legata alla retta all'infinito: per conseguenza, dal punto di vista proiettivo, essa non è che geometria del quadrilatero (o del quadrangolo) piano. Analogamente può dirsi che la metrica del tetraedro è caso particolare di quella del pentaedro, o del pentagono gobbo. 
nello spazio ordinario, del pentagono nẹllo spazio a 4 dimensioni, ecc. La cfz. $^{\text {ne }}$ comincia a essere interessante per $n=r+2$ perchè allora esistono gli spazi diagonali e principalmente perchè interviene in questo caso la considerazione veramente feconda del gruppo di $n$ ! collineazioni che permutano in tutti i modi possibili $i$ vertici del poligono. Proseguendo ad aumentare il numero dei vertici senza aumentare il numero delle dimensioni dello spazio ambiente, si può prevedere che l'interesse della ricerca abbia (in generale) a diminuire perchè il gruppo suddetto manca. Il caso più interessante è dunque dato da $n=r+2$. Ecco perchè nello spazio a 4 dimensioni si presenta promettente lo studio dell'esagono (*). Esso fu già intrapreso da Rrchmond in due lavori (**) hei quali però manca la considerazione e l'analisi del gruppo totale delle collineazioni che trasformano l'esagono in se stesso e che costituiscono la sorgente più viva delle proprietà geometriche inerenti alla cfz.

Lo scritto presente è indipendente dalle ricerche di Rrchmono e (come ormai è costume) accennerò a qualche punto rilevante contenuto nelle pagine seguenti che lo compongono.

Prima di tutto è chiaro che in $S_{4}$ lo studio dell'esagono è identico allo studio della cfz. composta da sei spazi, a tre dimensioni lineari, in posizione generica e che ho chiamato esaedro. Basta attuare la trasformazione polare rispetto alla quadrica invariante ( $\left(.^{\circ} 2\right)$ per passare dall'esagono all'esaedro e viceversa. Cosicchè si può scegliere come cfz. iniziale l'uno, o l'altro indifferentemente, come pure si potrà riferire all'uno, o all'altro il linguaggio relativo ad ogni resultato. Però, nella scelta, ho aruto di mira che ogni teorema fosse il più espressivo possibile dal punto di vista della intuizione geometrica e il servirsi dell'esagono, o dell'esaedro può mutare sapore alla frase pur mantenendone la sostanza. La geometria dell'esagono in $S_{4}$ ha

(*) I casi precedenti sono ben conosciuti. Per $r=3$ si ha la cfz. del pentagono, o del pentaedro, nello spazio ordinario, sulla quale lo scritto più recente è la mia Memoria che si intitola dal pentaedro (cf. Circ. Mat. Palermo, T. XXI, 1906, pp. 322-341) e dalla quale si può avere notizia dei lavori anteriori. Per $r=2$ la $\mathrm{cf} z$. ̀̀ quella del quadrangolo o del quadrilatero piano. L'ultima pubblicazione in argomento è di Berzol.ARI, porta il titolo: La lemniscata proiettiva (Rendic. Istit. Lomb, s. II, Vol. XXXVIII, 1904, pp. 277-288 e pp. 304-313). Finalmente il caso $r=1$ è realizzato da una terna di punti su di una retta, con la forma $Q$, con il comune gruppo hessiano, ecc.

(*) H. W. RICHмond, On the figure of six points in space of four dimension. Quarterly, n. $.^{\circ} 122,1899, \mathrm{pp} .125-160$. - The figure formed from six points in space of four dimension. Math. Annal., Bd. 53, pp. 161-176.

Annali di Matematica, Serie III, Tomo XVI. 
delle analogie con quella dell'esagrammo mistico. Ne è causa un fatto comune ad entrambe le teorie. Come ogni esagono ordinato di un esagrammo mistico individua la propria retta di PAscas, così (in modo non uguale, ma analogo) ogni esagono ordinato di un esagono completo in $S_{4}$ individua, mediante la considerazione delle 3 coppie di lati opposti, una retta che ho chiamato ancora retta di PASGaL per ragione dell'analogia suddetta (*). La quale dà motivo a credere di affermarsi con altri fátti. Ad esempio ai 20 punti di Steiner in cui concorrono a 3 , a 3 le 60 rette di Pascal dell'esagrammo mistico fanno riscontro 20 rigate quadriche a due a due coniugate su cui si distribuiscono a tre, a tre le 60 rette di PASGal dell'esagono completo di $S_{4}$, ecc., ecc.

Ho destinato un capitolo alla interpretazione geometrica di tutti i sottogruppi conosciuti del $G_{\tau 20}$ totale enunciando per ciascuno i fatti geometrici più salienti. Fra essi i più interessanti sono i sei $G_{120}$ : ciascuno funziona come gruppo totale sopra un noterole aggruppamento di cinque rette scelte fra le 15 diagonali di $1 .^{a}$ specie le quali dànno luogo così a sei tali aggruppamenti. Del resto queste 15 rette, oppure i 15 piani duali, furono già considerati da altri autori. Chi vuol prenderne notizia precisa legga le belle pagine che il prof. Bentrix dedica nel suo libro iperspaziale alla $V_{3}^{3}$ di $S_{4}$ luogo delle rette che si appoggiano a 4 piani generici (**).

Ho anche considerato le ipersuperficie invarianti rispetto a $G_{\tau 20}$ e d'ordine inferiore a sei. Non esiste alcuno spazio lineare invariante. Esiste una sola quadrica (alla quale abbiamo già accennato): anche le ipersuperficie cubiche invarianti si riducono a una sola che è la $V_{3}^{3}$ dianzi nominata e che nel seguito noi indicheremo col nome del SEGRE $\left(^{* *}\right)$ dal primo che l'ha considerata. Esistono finalmente infinite ipersuperficie invarianti di $4 .^{\circ}$ e $5 .^{\circ}$ ordine: tanto le une quanto le altre formano ún fascio. Fra le quartiche è da segnalare la $W$ di Castelnovo (****) dotate di 10 rette doppie distribuite a sei,

(*) Anche Richmond considera 60 rette collegate alla cfz. che egli chiama rette di PAScAt, ma non apparisce affatto il legame di ciascuna con un esagono ordinato: esse dipendono da altre considerazioni e in ogni modo sono differenti dalle mie.

(**) E. Bertini, Introduzione alla geometvia proiettiva degli iperspazi, pp. 176-177. Pisa, Spoerri, 1907.

(**) SEgre, Sulla varietà cubica con 10 punti doppi (Atti R. Acc. di Torino, 29, 1887) e Sulle varietà cubiche, ecc. (Mem. id. 39 (2), 1888).

${ }^{(* * * *)}$ Castelixovo, Ricerche di Geometria della retta nello spazio a 4 dimensioni (Atti R. Istit. Veneto, T. II, s. VII, .pp. 855-901). 
a sei sopra 10 quadriche (tre su ciascuna serie rigata). Fra le quintiche la più notevole appare la Hessiana della $V_{3}^{3}$ di SEcrie, a causa delle sue linee e punti singolari, così da far ritenere possibile di utilizzarla per studiarne opportune sezioni con spazi a tre dimensioni, nella speranza di acquisire qualche esempio di superficie quintica del nostro spazio che sia nuovo e interessante dal lato proiettivo per il numero e la posizione dei punti singolari. Del resto, non è questo il solo argomento del presente scritto che sembri meritare ancora qualche considerazione ulteriore: anche limitandosi a cercare risultati inerenti alla geometria del nostro spazio rimangono a considerarsi le proiezioni e le sezioni più opportune delle cfz. qui studiate. Un cosiffatto indirizzo di ricerche costituisce lo scopo principale delle già citate Memorie di Richmond, ma esse non esauriscono certo l'argomento in quistione.

\section{CAPITOLO I.}

\section{Gli elementi fondamentali dell'esaedro e del suo esagono polare.}

1. - Lo spazio ambiente sia lineare ed abbia quattro dimensioni. Per indicarlo lo chiameremo per brevità «spazio ambiente» senz'altro. Esso contiene punti, rette, piani e spazi lineari a tre dimensioni che saranno chiamati spazi senz'altra qualifica. Nello spazio ambiente consideriamo sei spazi in posizione generica e chiamiamo esaedro la cfz. ${ }^{\mathrm{n}}$ a cui essi dànno luogo. Indicheremo con $S(1), S(2), \ldots, S(6)$ tali spazi e li chiameremo gli «spazi fondamentali » dell'esaedro.

Riferendo un punto qualunque $M$ dello spazio ambiente a questi sei spazi indicheremo con $x_{1}, x_{2}, \ldots, x_{6}$ le coordinate di $M$ (con la condizione $\left.\sum_{1}^{6} x_{i}=0\right)$ e allora le equazioni $x_{1}=0, x_{2}=0, \ldots, x_{6}=0$ serviranno a rappresentare analiticamente i sei spazi fondamentali suddetti. Due spazi fondamentali come $S(1), S(2)$ si tagliano secondo un piano che chiameremo « faccia» dell'esaedro e indicheremo col simbolo $S(12)$ : Esso è rappresentato analiticamente da $x_{1}=0, x_{2}=0$. L'esaedro ha dunque 15 facce. Ogni spazio 
fondamentale ne contiene $\check{5}$. Tre spazi fondamentali come $S(1), S(2), S(3)$ hanno in comune una retta che chiameremo "spigolo" dell'esaedro e indicheremo col simbolo $S(123)$. Essa è rappresentata analiticamente da $x_{1}=0$, $x_{2}=0, x_{3}=0$. L'esaedro ha dunque 20 spigoli: ogni faccia ne contiene 4 . Finalmente 4 spazi fondamentali come $S(1), S(2), S(3), S(4)$ hanno in comune un punto che sarà chiamato "vertice » dell'esaedro è indicato col simbolo $S$ (1 234). Le sue coordinate sono dunque (00001-1). L'esaedro ha quindici vertici: ogni spigolo ne contiene tre. Sopra ogni spazio fondamentale gli altri cinque individuano un pentaedro completo. Si hanno così sei pentaedri completi di cui i vertici, gli spigoli e le facce sono rispettivamente vertici, spigoli e facce dell'esaedro.

2. - Una permutazione qualsiasi operata sopra i sei spazi fondamentali individua una collineazione nello spazio ambiente che trasforma l'esaedro in se medesimo. Abbiamo dunque così un gruppo di 720 collineazioni che indicheremo con $G_{720}$ e rispetto al quale l'esaedro è invariante. Tali collineazioni possono rappresentarsi analiticamente mediante le permutazioni sulle $x_{i}$. A suo tempo studieremo questo gruppo con qualche dettaglio. Ma fin d'ora osserveremo che esso non possiede alcun spazio lineare invariante, gìachè l'equazione che lo rappresenterebbe dovrebbe essere lineare e simmetrica nelle $x_{i}$; essa sarebbe dunque la $\sum_{1}^{6} x_{i}=0$ la quale non definisce nessuno spazio perchè ad essa soddisfano, per ipotesi, le coordinate di qualunque punto dello spazio ambiente. Esiste invece una ed una sola quadrica invariante, non specializzata, che può rappresentarsi indifferentemente con l'una o con l'altra delle due equazioni

$$
\sum_{1}^{6} x_{i}^{2}=0 ; \quad \sum_{1}^{6} x_{i} x_{k}=0
$$

a causa di $\sum_{1}^{6} x_{i}=0$. Qualificheremo questa quadrica con l'aggettivo «invariante» e la indicheremo col simbolo $F$. Si può dunque dire:

* Esiste una sola quadrica (a tre dimensioni) invariante rispetto al gruppo $G_{720}$ totale: essa taglia ogni spazio fondamentale dell'esaedro secondo la quadrica ( $a$ due dimensioni) che è invariante rispetto a quel soltogruppo $G_{120}$ che tiene fisso quello spazio $\left({ }^{*}\right)$ : taglia ogni faccia dell'esaedro secondo la conica

(*) CIANI, loc. cit., n. ${ }^{\circ} 2$. 
invariante rispetto a quel sottogruppo $G_{24}$ che tiene fissa quella faccia $\left(^{*}\right)$ e finalmente taglia ogni spigolo dell'esaedro nella coppia Hessiana dei tre vertici situati su quello spigolo (la quale coppia Hessiana è invariante rispetto al sottogruppo $G_{6}$ che tiene fisso tale spigolo)».

3. - Ebbene: l'esaedro con i suoi spazi fondamentali, con le sue facce, i suoi spigoli e vertici compone una cfz." la quale individua la sua polare reciproca rispetto alla quadrica $F$ suddetta. Si perviene così ad un esagono i cui vertici, spigoli, facce e spazi fondamentali sono polari reciproci rispettivamente degli spazi fondamentali, delle facce, degli spigoli e dei vertici dell'esaedro dato.

I vertici dell'esagono sono sei: le coordinate del vertice polo di $S(1)$ sono $(-5,1,1,1,1,1)$ : ed è ovvio scrivere le coordinate degli altri cinque. Indicheremo con $\Sigma(i)$ questi vertici.

Gli spigoli dell'esagono sono 15 . Indicheremo con $\Sigma(i k)$ lo spigolo che congiunge $\Sigma(i)$ con $\Sigma(k)$ : esso è polare di $S(i k)$. Lo spigolo $\Sigma(12)$ può essere rappresentato dalle equazioni simultanee $x_{3}=x_{4}=x_{5}=x_{6}$ (che si riducono a tre indipendenti).

Le facce dell'esagono sono 20 . Indicheremo con $\Sigma(i h k)$ la faccia individuata da $\Sigma(i), \mathbf{\Sigma}(h), \mathbf{\Sigma}(k)$. Essa è polare di $S(i \hbar k)$. La faccia $\Sigma(123)$ è rappresentata analiticamente dalle equazioni $x_{4}=x_{5}=x_{6}$ (che si riducono a due indipendenti).

Finalmente l'esagono ha 15 spazi fondamentali. Indicheremo con $\Sigma(i h k l)$ lo spazio individuato da $\Sigma(i), \Sigma(h), \Sigma(k), \Sigma(l)$. Esso è il polare di $S(i h k l)$. Lo spazio $\Sigma(1234)$ è rappresentato dalla equazione $x_{3}=x_{6}$.

È poi evidente che essendo generico l'esaedro, anche l'esagono polare è generico e si potrebbe manifestamente partire dall'esagono per definire l'esaedro. Per cui tutta la cfz. ${ }^{\text {ne }}$ che ci proponiamo di considerare ammette per così dire come nuclei di origine l'esaedro e l'esagono suddetti polari reciproci rispetto alla quadrica $F$. Qualificheremo l'uno e l'altro con l'aggettivo "fondamentale».

(*) Berzolari, loc. cit., n. ${ }^{\circ} 1$. 


\section{CAPITOLO II.}

\section{Gli elementi diagonali.}

4. - Combinando in vario modo gli elementi fondamentali dell'esaedro e dell'esagono si ottengono punti, rette, piani e spazi i quali chiameremo «diagonali» per analogia a elementi così chiamati e inerenti al quadrangolo e quadrilatero del piano, e al pentaedro e pentagono dello spazio a tre dimensioni. Se non che nel caso dell'esaedro e dell'esagono attuali questi elementi sono tanti e tali da consigliare una scelta. Noi presenteremo al lettore quelli che sembrano i più notevoli in riguardo a considerazioni ulteriori contenute nel presente scritto. Intanto le definizioni che siamo per dare sono legate al concetto di elementi «opposti». Un vertice e una faccia dell'esaedro si diranno opposti quando la faccia è comune ai due spazi fondamentali che non passano per il vertice. Due spigoli dell'esaedro si diranno opposti quando non esistono in un mędesimo spazio fondamentale. Ebbene lo spazio individuato da un vertice e dalla faccia opposta si chiamerà « spazio diagonale di 1." specie». Così ad es. $S\left(\begin{array}{lll}1 & 234\end{array}\right)$ e $S(56)$ individuano lo spazio diagonale di $1 .^{\mathrm{a}}$ specie rappresentato da $x_{5}+x_{6}=0$ e che indicheremo con $\Delta(56)$. Esistono 15 tali spazi. Analogamente: lo spazio individuato da due spigoli opposti dell'esaedro come $S\left(\begin{array}{lll}1 & 23\end{array}\right), S\left(\begin{array}{l}456) \\ 5\end{array}\right)$ sarà chiamato «spazio diagonale $d i 2 .^{*}$ specie» $\mathrm{e}$ indicato con l'uno o l'altro dei due simboli equivalenti $\Delta(123)$, ovvero $\Delta(456)$. L'equazione è $x_{1}+x_{2}+x_{3}=0$, ovrero la sua equivalente $x_{4}+x_{5}+x_{6}=0$. Esistono 10 tali spazi. Finalmente chiameremo «spazi diagonali di 3." specie» quelli rappresentati da equazioni del tipo $x_{1}+x_{2}=x_{3}+x_{4}$. II simbọlo relativo sarà $\Delta(12,34)$. Esistono 45 tali spazi.

5. - Chiameremo punti diagonali gli elementi polari dei precedenti rispetto alla quadrica $F$. Cosi, premessa la definizione di elementi opposti dell'esagono (dualmente a quella data innanzi per l'esaedro), si chiamerà

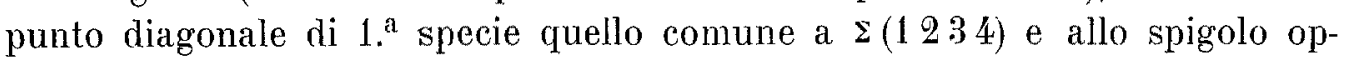
posto $\Sigma(56)$. Indicheremo con $D(56)$ un tale punto: le sue coordinate sono (1 $11111-2-2)$.

I punti diagonali di prima specie sono 15. 
Prese invece due facce opposte dell'esagono, come $\Sigma(123), \Sigma(456)$ si chiamerà "punto diagonale di 2." specie" il loro punto comune. Sarà indicato con l'uno, o l'altro dei due simboli equiralenti $D(123)$, orvero $D(456)$. Le coordinate sono $(1,1,1,-1,-1,-1)$. Esistono 10 punti diagonali di 2. ${ }^{\text {a }}$ specie.

Finalmente: esistono 45 punti diagonali di $3 .^{a}$ specie. Il simbolo è $D(i h, k l)$, e ad esempio $D(12,34)$ ha per coordinate $(1,1,-1,-1,0,0)$.

Sono così accoppiate tre specie di spazi diagonali e tre specie di punti díagonali secondo la polarità rispetto alla quadrica $F$. I simboli rispettivi sono caratterizzati dalle lettere $\Delta$ e $D$.

6. - In modo analogo accoppieremo ora piani e rette diagonali caratterizzando i primi col simbolo $\delta$ e le seconde col simbolo $d$ e distinguendo tre specie.

Chiameremo "piani diagonali di 1." specie" quelli individuati da equazioni del tipo $x_{1}+x_{2}=0, x_{3}+x_{4}=0, x_{5}+x_{8}=0$, notando bene che tali equazioni si riducono a due indipendenti a causa di $\sum_{1}^{6} x_{i}=0$. Il simbolo corrispondente sia $\delta(12,34,56)$. Esistono 15 tali piani.

Chiameremo piani diagonali di 2. ${ }^{a}$ specie quelli rappresentati da equazioni del tipo $x_{1}=x_{2}, x_{3}=x_{4}$; cioè la intersezione di due spazi fondamentali dell'esagono aventi due vertici comuni. Il simbolo relativo sia $\delta(12,34)$. Essi sono in numero di 45 . E finalmente chiameremo «piani diagonali di 3." specie» quelli rappresentati da equazioni del tipo $x_{1}=0, x_{2}+x_{3}=0$. Il simbolo sia $\delta(1,23)$. Essi sono 60 . Gli elementi polari rispetto a $F$ delle tre specie di piani diagonali ora descritti si chiameranno ordinatamente « rette diagonali di $1 .{ }^{\prime \prime}, 2 . ", 3 .{ }^{\prime \prime}$ specie indicandole con i simboli rispettivi : $d(i h$, $k l, m n) ; d(i h, k l) ; d(i, h k)$. Si hanno dunque 15 rette diagonali di $1 .^{\text {a }}$ specie; 40 di $2 .^{a}$ e 60 di $3 .^{a}$

7. - $\grave{\mathrm{E}}$ utile avvertire che fra gli elementi diagonali ora introdotti figurano tutti quelli che con questo nome si presentano nei sei pentaedri fondamentali compleli di cui è parola alla tine del $n^{0}{ }^{0}$. Adottando le denominazioni della mia Memoria sul pentaedro si vede che $\mathrm{i}$ piani, le rette e $\mathrm{i}$ punti diagonali dei sei pentaedri suddetti sono ordinatamente: i piani diagonali di 3..$^{a}$ specie, le rette diagonali di $2 .^{a}$ e i punti diagonali di $3 .^{a}$ della cf ${ }^{\text {ne }}{ }^{\text {at }}$ attuale.

Nel quadro della pagina seguente sono riuniti per comodità del lettore gli elementi diagonali sin qui descritti. 


\section{ELEMENTI LINEARI DELLA GONFIGURAZIONE DIAGONALE}

\section{Polaritì RISPETTO ALLA QUADRIGA $F$.}

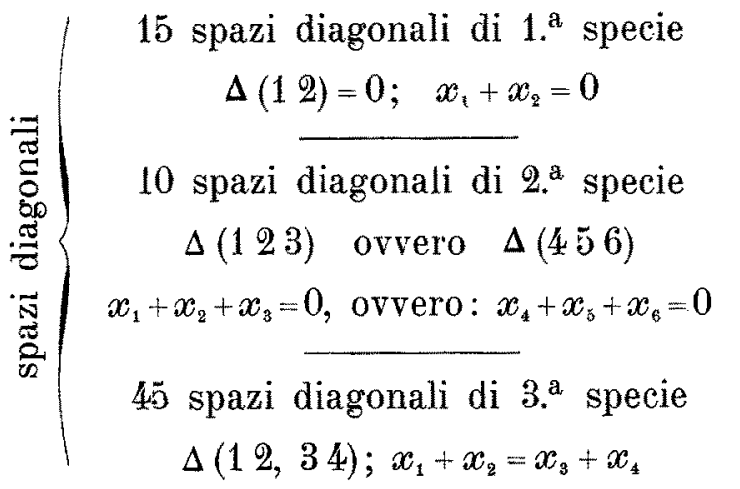
15 piani diagonali di $10^{\mathrm{a}}$ specie $\delta(12,34,56)$

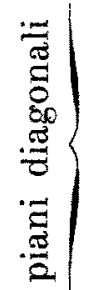
$\left(x_{1}+x_{2}=0, x_{3}+x_{4}=0, x_{5}+x_{6}=0\right)$
45 piani diagonali di 2. ${ }^{\mathrm{a}}$ specie $\delta(12,34) ;\left(x_{1}=x_{2}, x_{3}=x_{4}\right)$
60 piani diagonali di $3 .^{a}$ specie $\delta(1,23) ;\left(x_{1}=0, x_{2}+x_{3}=0\right)$

NB. : $\Delta$ è simbolo di spazio diagonale $d$ » pianó
15 punti diagonali di $1 .^{a}$ specie

$$
D(12) \equiv(-2,-2,1,1,1,1)
$$

10 punti diagonali di $2 .^{a}$ specie

$$
D(123) \text { ovvero } D(456)
$$$$
\equiv(1,1,1,-1,-1,-1)
$$

45 punti diagonali di $3 .^{a}$ specie $D(12,34) \equiv(1,1,-1,-1,0,0)$

15 rette diagonali di $1 .^{\mathrm{a}}$ specie

$$
d(12,34,56)
$$

$(1+\lambda, 1+\lambda,-1,-1,-\lambda,-\lambda)$

45 rette diagonali di $2 .^{\mathrm{a}}$ specie

$$
\begin{gathered}
d(12,34) \\
(\lambda,-\lambda, 1,-1,0,0)
\end{gathered}
$$

60 rette diagonali di $3 .^{\mathrm{a}}$ specie

$$
d(1,23)
$$

$(-5+\lambda, 1-2 \lambda, 1-2 \lambda, 1+\lambda, 1+\lambda, 1+\lambda)$

$N B .: \lambda$ è un parametro

$\ldots D$ è simbolo di punto diagonale $\ldots d$ » 


\section{CAPITOLO III.}

\section{La configurazione diagonale.}

8. - Ci proponiamo ora di mettere in rilievo i più notevoli legami geometrici che vincolano fra di loro gli elementi diagonali introdotti nel capitolo precedente. Intanto cominceremo dal notare come, mediante la considerazione degli spazi diagonali di $1 .^{\text {a }}$ specie, si possa, dall'esaedro, costruire facilmente l'esagono, senza ricorrere alla polarilà rispetto alla quadrica invariante. Basta semplicemente osservare che i due spazi fondamentali dell'esaedro, passanti per una faccia qualunque, sono separati armonicamente dallo spazio diagonale di $1 .^{\mathrm{h}}$ specie e dallo spazio fondamentale dell'esagono passanti per quella medesima faccia. Un tale gruppo, armonico, è ad es. $S(1)$, $S(2), \Delta(12), \Sigma(3456)$. Ecco dunque come si può descrivere la costruzione cercata :

"Dato l'esaedro, si osservi che per una sua faccia qualunque passano due spazi fondamentali e uno spazio diagonale di 1." specie. Ebbene, si costruisca, per quella faccia, lo spazio coniugato armonico dello spazio diagonale rispetto ai due fondanentali suddetti, e si ripeta questa costruzione per tutte le facce suddette, Si troveranno cosi 15 spazi $i$ quali vengono a passare a 10, a 10 per ser punti vertici dell'esagono polare richiesto. »

9. - Consideriamo una retta diagonale di $1 .^{a}$ specie come ad es. la $d(12,34,56)$. Dalla rappresentazione parametrica risulta immediatamente che essa contiene i 6 punti $D(12), D(34), D(56) ; D(12,34), D(12,56)$; $D(34,56)$ e che $D(12)$ è coniugato armonico di $D(34,56)$ rispetto alla coppia $D(34), D(56)$, ecc. Dunque si può dire:

"Ogni retta diagonale di 1." specie contiene sei punti diagonali e cioè tre di 1." specie e tre di 3." specie. Queste due terne si deducono l'una dall'altra costruendo il quarto armonico di un punto dell'una rispetto ai due rimanenti. Servendosi di un linguaggio ben noto si può dire che le due terne sono ciascuna la forma $Q^{\circ}$ dell'altra $\left.{ }^{*}\right)$. 11 comune gruppo Hessiano è costituito dalla coppia di punti d'incontro della retta diagonale in parola con la quadrica $F$."

(*) Cf. p. es. Clebsch-Lindemann. Vol. I, pag. 275. 
10. - Nello spazio ambiente è chiaro che tre rette in posizione generica ammettono una sola trasversale comune. Si considerino allora tre lati dell'esagono fondamentale non aventi a due, a due in comune alcun vertice: ad es. $\Sigma(12), \Sigma(34), \Sigma(56)$. Sopra $\Sigma(12)$ esiste $D(12)$; sopra $\Sigma(34)$ esiste $D(34)$; sopra $\Sigma(56)$ esiste $D(56)$; ma $D(12), D(34), D(56)$ esistono a loro volta sopra $d(12,34,56)$ secondo il numero precedente. Tale trasversale è quindi precisamente $d(12,34,56)$. Si ha dunque il risultato:

"I lati dell'esagono possono raggrupparsi in quindici terne così che $i 3$ lati di ciascuna terna non abbiano a due, a due vertici comuni: ogni terna individua una ed una sola trasversale comune alle rette della terna: si trovano, in tal guisa, 15 trasversali che costituiscono le 15 diagonali di $1 . "$ specie. I punti di appoggio di esse con $i$ lati suddetti, sono $i 15$ punti diagonali di $1^{*}$ specie."

11. - Per il punto $D(12)$ passano $a(12,34,56) ; d(12,36,45)$; $d(12,35,46)$ le quali esistono tutt'e tre in $\Sigma(3456)$. D'altra parte $d(12$, $34,56)$ contiene $D(12), D(34), D(56)$. Si ha perciò : " $I 15$ punti e le 15 rette diagonali di $1^{.}$specie compongono una of $z .\left(15_{3}, 15_{3}\right)$ cioè tale che ogni relta contiene tre punti e per ogni punto passano 3 rette. Le 3 rette della cfz. che passano per un punto esistono in uno spazio fondamentale dell'esagono."

12. - Uno spazio diagonale di $2 .^{\mathfrak{a}}$ specie, come $\Delta(123)$, contiene sei rette diagonali di $10^{a}$ specie che noi divideremo nelle due terne seguenti:

$$
\begin{aligned}
& d(14,25,36) ; \quad d(15,26,34) ; \quad d(16,24,35) \\
& d(14,26,35) ; \quad d(15,24,36) ; \quad d(16,25,34) \text {. }
\end{aligned}
$$

$\grave{E}$ facile constatare che due di tali 6 rette s'incontrano, o no, a seconda che appartengono, o no, a terne differenti. Quindi :

"Ogni spazio diagonale di 2." specie contiene sei relte diagonali di $1^{*}$ specie le quali si dividono in due terne appartenenti a due serie rigate coniugate su di una medesima quadrica.»

Si hanno così 10 di queste quadriche (a due dimensioni). Le indicheremo con $Q(i h k)$. Sopra $Q(123)$, o $Q(456)$ (che è lo stesso) esistono quelle sei rette $d(i h, k l, m i n)$ i cui simboli binari $i h, k l, m n$ portano ciascuno uno uno dei tre simboli $1,2,3$ e uno dei tre $4,5,6$.

13. - Un piano diagonale di $3 .^{\mathrm{a}}$ specie come $\delta(1,23)$ contiene i sei punti diagonali di $3 .^{\mathrm{a}}$ specie seguenti :

$$
D(25,36) ; D(26,35) ; D(24,36) ; D(26,34) ; D(24,35) ; D(25,34) \text {. }
$$


D'altra parte tale piano è anche diagonale per il pentaedro che viene individuato sopra $S(1)$ dagli altri 5 spazi fondamentali dell'esaedro e i 6 punti precedenti sono diagonali pure per il pentaedro e quindi esistono su di una conica (cf. la mia Memoria «sul pentaedro»n. ${ }^{\circ} 18$ ). Dunque:

«I 45 punti diagonali di $3 .^{*}$ specie esistono $a 6, a 6$ sopra 60 coniche giacenti nei 60 piani diagonali di $3 .^{\circ}$ specie.»

Una diagonale di $2 .^{a}$ specie come $d(12,34)$ contiene i due punti diagonali di $3 .^{a}$ specie seguenti: $D(13,24), D(14,23)$ e i due vertici dell'esaedro $S(1256), S(3456)$. Finalmente una diagonale di $3 .^{a}$ specie come $d(1,23)$ contiene $\Sigma(1)$, che è un vertice dell'esagono fondamentale, e il punto $D(23)$ che è diagonale di $1 .^{\text {a }}$ specie.

\section{GAPITOLO IV.}

\section{Le 60 rette di Pascal dell'esagono fondamentale.}

14. - Come già abbiamo dichiarato nelle brevi pagine che hanno servito a guisa di introduzione al presente scritto, l'esagono fondamentale individua 60 rette inerenti ai suoi 60 esagoni ordinati che hanno analogia con le 60 rette di PASCAL dell'esagrammo mistico. E l'analogia è tale da ritenere opportuno di conservare la denominazione. Ecco come possono presentarsi queste rette: indichiamo con $\Sigma(123456)$ l'esagono ordinato i cui vertici si susseguono nell'ordine indicato dalla permutazione circolare chiusa in parentisi. Poi si osservi che presi due lati opposti come $\Sigma(12), \Sigma(45)$ e la retta che passa per i vertici rimanenti, cioè la $\Sigma(36)$, esiste la diagonale di $1 .{ }^{a}$ specie $d(12,45,36)$ appoggiata a tutte e tre le rette suddette $\left(n .^{\circ} 10\right)$ essendo $D(12), D(45), D(36)$ i punti di appoggio. Dopo di che si costruisca il coniugato armonico di $D(36)$ rispetto a $D(12), D(45)$ ossia $\left(n .^{\circ} 9\right)$ il punto $D(12,45)$. Così dai lati opposti $\Sigma(12), \Sigma(45)$ si è pervenuti a costruire $D(12,45)$. In ugual modo dalle altre due coppie di lati opposti si perviene a $D(23,56), D(34,61)$. Ebbene dico che i tre punti:

$$
D(12,45) ; \quad D(23,56) ; \quad D(34,61)
$$


sono in linea retta che sarà chiamata retta di PAşCAL del dato esagono ordinato. Per dimostrare questa affermazione basta ricorrere alla collineazione $(135)(246)$ la quale subordina in $\Delta(123)$ una collineazione biassale a periodo tre (cf. il $n^{\circ} 20$ ) e poi osservare che $i$ tre punti suddetti esistono in $\Delta(123)$ e ivi compongono un ciclo: essi dunque stanno su di una retta (unita per la collineazione nominata). Questa retta è vincolata al considerato esagono ordinato $\Sigma(123456)$ e la indicheremo col simbolo $p(123456)$.

Esistono dunque 60 rette di Pascal in tutto.

Riunendo allora il resultato attuale con quello del $n .^{\circ} 10$ si può enunciare il teorema seguente:

«I 45 punti diagonali di 3." specie giacciono a 3, a 3 sopra 75 rette: 15 di esse sono le 15 diagonali di 1." specie, le 60 rimanenti sono le 60 rette di PASCAL dei 60 esagoni ordinali contenuti nell'esagono completo fondamentale. »

15. - Quand'è che due rette di PASGal s'incontrano? Ciò accade, manifestamente, quando gli esagoni ordinati relativi hanno una coppia comune di lati opposti, chè allora il punto comune alle due rette è diagonale di $3 .^{a}$ specie. Ma possono anche incontrarsi in un altro caso: basta che $\mathrm{i}$ due esagoni abbiano in comune una coppia di vertici opposti e $\mathrm{i}$ lati concorrenti in questi vertici. .Così s'incontrano

$$
p(123456), \quad p(123654)
$$

e le coordinate del punto comune sono $(1,2,1,-1,-2,-1)$. Esso è, sulla prima, il coniugato armonico di $D(34,61)$ rispetto a $D(12,45)$ e $D(23,56)$ e, sulla seconda, il coniugato armonico di $D(36,14)$ rispetto a $D(12,56), D(23,54)$. Ne segue che la $p(123456)$ incontra, fuori dei punti diagonali di $3 .^{a}$ specie le altre tre rette seguenti:

$$
p(123654), \quad p(143256), \quad p(163452) \text {. }
$$

D'altra parte per $D(12,45)$ passano le 4 rette seguenti:

$$
p(123456), p(126453), \quad p(123546), \quad p(126543) \text {. }
$$

Dunque: «Una retta di PASCAL ne incontra altre 12. Di queste, 9 sono incontrate (a tre, a tre) nei 3 punti diagonali di 3." specie esistenti sulla retta nominata: le tre rimanenti sono incontrate nei tre punti coniugati armonici di ciascuno dei precedenti rispetto agli altri due."

16. - Tenendo conto di questi resultati si vede che le due terne 
seguenti

$$
\begin{aligned}
& p(123456) ; \quad p(143652) ; p(163254) \\
& p(123654) ; \quad p(143256) ; p(163452)
\end{aligned}
$$

appartengono a due serie rigate coniugate di una medesima quadrica che è $Q\left(\begin{array}{ll}135 \\ 3\end{array}\right)$ (cf. il n. ${ }^{\circ}$ 12). I sei esagoni relativi sono quelli che hanno per vertici alternati $135 ; 246$. Si ha quindi :

"Le 60 relte di PAscal appartengono a 6, a 6 alle stesse quadriche $Q(i h k)$ a cui appartengono pure a 6 , a 6 le 15 diagonali di 1." specie e, come quest'ultime, su ciascuna di tali quadriche esse si dividono in due terne appartenenti alle due serie rigate coniugate. »

Per ogni diagonale di $1 .^{\mathrm{a}}$ specie, come $d(12,34,56)$, passeranno 4 quadriche $Q(i h k)$ e cioè $Q(135), Q(136), Q(145), Q(146)$. Invece per ogni retta di PAScal, come $p(123456)$ passa la sola $Q(135)$.

17. - Esistono 4 esagoni che hanno i medesimi vertici opposti 14 , 25, 36. Le rette di Pascal sono le seguenti:

$$
\begin{aligned}
& p(123456) \text { che contiene: } D(12,45), D(23,56), D(34,61) \\
& p(153426) \quad \gg \quad \gg \quad D(15,42), D(53,26), D(34,61) \\
& p(126453) \quad \text { » } \quad D(12,45), D(26,53), D(64,31) \\
& p(156423) \quad \text { » } \quad D(15,49), D(56,23), D(64,31)
\end{aligned}
$$

quindi queste rette e questi punti sono lati e rertici di un quadrilatero completo esistente in $\delta(14,25,36)$, cioè :

"Le 60 rette di Pascal esistono $a \dot{f}$, a 4 nei 15 piani diagonali di $1^{*}$ specie: il quadrilatero che esse compongono, in ciascuno di tali piani, ha per vertici i sei punti diagonali di 3." specie che quel piano contiene».

18. - La cfz. dell'esagono fondamentale procede manifestando altri punti di contatto con quella dell'esagrammo mistico. Così sarebbe facile vedere che i 20 punti di STErner dell'esagrammo hanno il loro riscontro nelle 20 serie rigate (coniugate a due, a due) delle 10 quadriche $Q(i h k)$. Ma l'indagine ulteriore di queste analogie ci porterebbe troppo fuori del cammino che ei siamo proposti. Piuttosto passeremo adesso ad esaminare la struttura delle collineazioni componenti il $G_{720}$ totale. 


\section{GAPITOLO V.}

\section{Le collineazioni componenti il $G_{720}$.}

19. - Descriveremo queste collineazioni classificandole secondo il valore numerico del loro periodo. Ogni collineazione decomporremo in cicli effettuati sugli spazi fondamentali dell'esaedro, e per semplicità scriveremo i cicli effettuati sugli indici $1,2,3,4,5,6$. Così ad esempio il simbolo (123) rappresenterà la collineazione che attua la permutazione circolare $S(1) S(2)$ $S(3)$ e lascia fissi $S(4), S(5), S(6)$.

Periodo uguale a 2. Esistono le tre specie seguenti:

$$
\text { (1 2), (1 2) (3 4), (1 2) (3 4) (5 6). }
$$

Ma prima di descriverle separatamente faremo la seguente osservazione che vale per tutte. Siano $S_{r}, S_{4-r-1}$ gli spazi di punti uniti (con $r<4$ ) e $P P^{\prime}$ due punti corrispondenti. Siccome il periodo è due, la retta $P P^{\prime}$ è unita e quindi ha un punto, $M$, comune con $S_{r}$ e uno, $N$, comune con $S_{1-i-1}$ in guisa che $M N P P^{\prime}$ è un gruppo armonico. Vale dunque la seguente costruzione che serve a trovare $P^{\prime}$ quando si conosca $P$. Si congiunga $S_{\text {r }}$ con $P$ mediante l' $S_{r+1}$ che li contiene e si determini il punto $M$ comune a tale $S_{r+1}$ e all' $S_{t-r-1}$ : sia $N$ il punto comune alla retta $M P$ e a $S_{r}$ (punto che

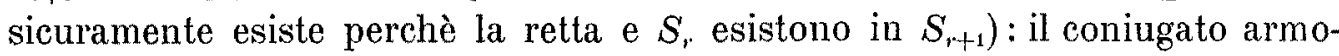
nico di $P$ rispetto ad $M N$ è il punto $P^{\prime}$ cercato.

Ciò premesso esaminiamo la $1 .^{\mathrm{a}}$ specie (12). Si ha $r=0$. 亡 punto unito $S(3456)$ è spazio di punti uniti $\Sigma(3456)$. La costruzione generica dianzi indicata, applicata a questo caso particolare, dimostra la opportunità di chiamare omologie armoniche le collineazioni di questa specie che sono 15. I centri di omologia sono i vertici dell'esaedro: gli spazi di omologia sono gli spazi fondamentali dell'esagono.

La $2 .^{\mathrm{a}}$ e la $3 .^{\mathrm{a}}$ specie si possono invece paragonare alle involuzioni gobbe del nostro spazio e noi lo chiameremo appunto con tale nome. Il luogo dei punti uniti della involuzione gobba $\left(\begin{array}{l}1 \\ 2\end{array}\right)\left(\begin{array}{l}3 \\ 3\end{array}\right)$ è costituito dalla retta diagonale di $2{ }^{\prime a}$ specie $d(12,34)$ e dal piano polare relativo $\delta(12,34)$. Le collineazioni di questa specie sono 45 . 
Invece il luogo dei punti uniti della (1 2) (34) (56) è composto della retta diagonale di $1 .^{\mathrm{a}}$ specie $d(12,34,56)$ e dal piano polare $\delta(12,34,56)$. Le collineazioni di questa specie sono $\mathbf{1 5}$.

20. - Periodo uguale a 3. Esistono le due specie seguenti :

$$
(123) ; \quad(123)(456) \text {. }
$$

Nella prima sono punti uniti tutti quelli di $\$(456)$ e i punti d'incontro di $S(456)$ con la quadrica $F$. Le collineazioni di questa specie sono 40 e a due, a due inverse l'una dell'altra.

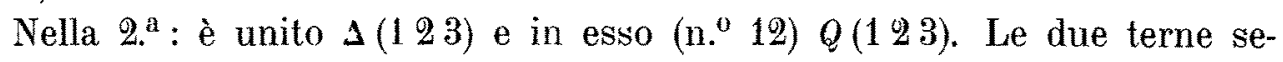
guenti : sopra $\left.Q\left(\begin{array}{lll}1 & 2 & 3\end{array}\right)\right)$

$$
\begin{aligned}
& d(14,26,35), \quad d(25,34,16), \quad d(36,15,24) \\
& d(14,25,36), \quad d(15,26,34), \quad d(16,24,35)
\end{aligned}
$$

sono unite, ma le rette della 1. a terna vengono permutate circolarmente, quelle della 2. sono invece mantenute fisse dalla collineazione in parola. Dunque si può dire che il luogo dei punti uniti è costituito dalle due rette che compongono la coppia Hessiana della prima terna e da $D(123)$, punto

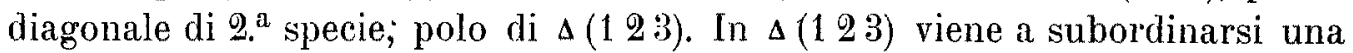
collineazione biassiale a periodo tre di cui gli assi sono le rette Hessiane suddette.

Le collineazioni di questa specie sono 40 (a due, a due inverse una dell'altra).

21. - Periodo uguale a 4. Si hanno le due specie:

$$
(1234) ; \quad(1234)(56) \text {. }
$$

Sono punti uniti della prima tutti quelli della retta $s(56)$ che è uno spigolo dell'esagono fondamentale: fuori di questa retta sono uniti i due punti d'incontro di $d(13,24)$ con la quadrica $F$ e il punto diagonale $D(13,24)$. Le collineazioni di questa specie sono 90 (a due, a due inverse).

Passiamo alla $2{ }^{\text {a }}$ specie:

$$
(1234)(56)
$$

Essa possiede la retta individuata dai due punti $D(135), D(136)$ come luogo di punti uniti. Fuori di questa retta è unito $D(56)$ e sono uniti i punti d'ineontro di $d(13,24)$ con $F$. 
Anche le collineazioni di questa specie sono 90 (a due, a due inverse). 22. - Periodo uguale a 5. Si ha una sola specie:

$$
\text { (1 } 2345) \text {. }
$$

È punto unito $\mathbf{\Sigma}(6)$. Nello spazio polare $S(6)$ si viene a subordinare una collineazione a periodo 5 che opera sul pentaedro completo le cui facce sono: $S(16), S(26), S(36), S(46), S(56)$. Valgono quindi le considerazioni del n. 11 della mia Memoria, più volte citata, sul pentaedro per la quale si vede che questa collineazione subordinata ha soli 4 punti uniti. Essi insieme a $\Sigma(6)$ costituiscono i cinque punti uniti della collineazione considerata.

II numero di queste collineazioni è 144 (a due, a due inverse).

23, - Periodo uguale a 6. Esistono le due specie:

$$
(123456) ;(123)(45) \text {. }
$$

La $1 .^{\text {a }}$ possiede 5 soli punti uniti che sono: $D(135)$ e i 4 punti d'incontro di $F$ con le due rette $d(14,25,36), p(123456)$, di cui la $1^{a}$ è diagonale di $1 .^{\mathrm{a}}$ specie e la $2{ }^{\mathrm{a}}$ retta di PASCA L dell'esagono ordinato 2 ( 123456 ).

Le collineazioni di questa specie sono 120 (a due, a due inverse).

Quanto alla 2. ${ }^{\mathrm{a}}$ si vede che è luogo di punti uniti la retta che passa

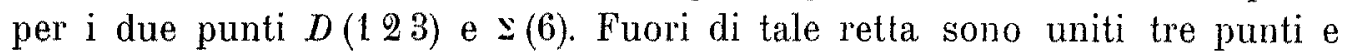
cioè $S(1236)$ e i punti d'incontro di $S(456)$ con $F$.

Anche le collineazioni di questa specie sono 120 (a due, a due inverse).

\section{GAPITOLO VI.}

\section{Descrizione dei sottogruppi di $G_{720}$ che non lasciano fisso alcun vertice dell'esagono (o alcuno spazio fondamentale dell'esaedro).}

24. - In questo capitolo ci proponiamo la descrizione di quanto è principalmente inerente ai sottogruppi di $G_{i 20}$. Tralasceremo però quelli di essi che lasciano fisso qualche spazio fondamentale dell'esaedro (e quindi qualche vertice dell'esagono) giacchè la rappresentazione geometrica di tali gruppi è ovvia o sulla retta, o sul piano, o nello spazio a tre dimensioni. 
Ci occuperemo dunque sistematicamente di quei sottogruppi che non lasciano fisso nessuno spazio fondamentale e li classificheremo a seconda dell'ordine, cominciando dagli ordini più bassi e salendo a mano, a mano, ai più alti. A tale scopo seguiremo la nota tabella di CAYLEY (*) tenendo conto delle correzioni apportateri da CoLE (**).

25. - Ordine uguale a 2. Esiste una sola specie $G_{2}^{\mathrm{I}}$ che è

$$
\left\{(12)(34)(56\}^{\prime} ; r=1,2 .\right.
$$

Il gruppo è ciclico: vale quindi il $n .^{\circ} 19$.

Il gruppo è dispari. Il sottogruppo pari massimo è l'identità. Il numero di questi gruppi è 15 .

26. - Ordine uguale a 3. Esiste una sola specie $G_{3}^{\mathrm{I}}$ che è

$$
\{(123)(456)\} ; r=1,2,3 .
$$

Anche questo è ciclico e quindi veggasi il n..$^{0} 20$.

Il gruppo è pari. Il numero è 20 .

27. - Ordine uguale a 4 . lisistono 4 specie che descriveremo separatamente.

$I^{\prime \prime}$ specie. $G_{4}^{\mathrm{I}}$ :

$$
\text { (1 2) (34) (56); (13) (24) (56); (14) (23); I }
$$

dove con $I$ si indica la identità.

Il gruppo si può generare con due qualsiasi delle sue prime tre collineazioni (fra quelle scritte). Sono punti invarianti tutti quelli della retta che unisce $D(145)$ con $D(146)$ e gli altri tre: $D(56), D(12,34), D(13,24)$. Il gruppo è dispari. È sottogruppo pari massimo $\{(14)(23)\}^{n} ; r=1$, 2. Il numero di questi gruppi è 45 .

$I I^{u}$ specie. $G_{4}^{\mathrm{II}}$ :

$$
\text { (1 2) (34) (56); (1 2) (34); (5 6); } 1 \text {. }
$$

Generando il gruppo con la $1 .^{\mathrm{a}}$ e la $3 .^{\mathrm{a}}$ collineazione si trova che sono invarianti tutti i punti delle due rette $d(12,34,56) ; d(12,34)$ e inoltre

(*) A. CAYLEY, On the substitution groups for two, three, four, five, six, seven and eight letters. The Quarterly Journal of Math., Vol. XXV, pp. 82-83. - Veggasi anche:

(**) 1. N. CoLt, Note on the substitution groups of six, seven and eight letters. Bulletin of the New-York Math. Soc. Vol. II, pp. 184-190 (1893). 
il punto $S(1234)$. Il gruppo è dispari. Il sottogruppo pari massimo è ( (1 2) $(34) r^{r} ; r=1,2$. Il numero è 45 .

III ${ }^{a}$ specie. $G_{i}^{\mathrm{III}}$ :

$$
\text { (12) }(34) ;(12)(56) ; \quad(34)(56) ; 1 .
$$

Generando il gruppo con la $1 .^{\mathrm{a}}$ e la $2 .^{\mathrm{a}}$ collineazione si vede subito che sono invarianti tutti i punti di $d(12,34,56)$, e inoltre $S(1234), S(1256)$, $S(3456)$. Il gruppo è pari. Il numero è 15 .

$I V^{a}$ specie. $G_{4}^{\mathrm{IV}}$ :

$$
\{(1234)(56)\} ; \quad r=1,2,3,4 .
$$

Il gruppo è ciclico. Veggasi dunque il n..0 21 .

Il gruppo è pari. Il numero è 45 .

28. - Ordine uguale a 6 . Esistono 3 specie.

$I^{a}$ specie. $G_{6}^{\mathrm{T}}$ :

$$
(123456)^{r} ; r=1,2,3,4,5,6 .
$$

Il gruppo è ciclico. Veggasi dunque il n. ${ }^{\circ} 23$. Il gruppo è dispari. 亡̀ sottogruppo pari massimo $G_{3}^{\mathrm{I}}$. Il numero è 60 .

$I I^{a}$ specie. $G_{6}^{\mathrm{II}}$ :

$$
\{(123)(456)\} ; \quad r=1,2,3) ; \quad(12)(45) ; \quad(23)(56) ; \quad(31)(64) \text {. }
$$

È invariante il solo punto $D(123)$. 11 gruppo è pari.

Il numero è 60 (triplo di quello dei $G_{3}^{\mathrm{I}}$ ).

$$
I I I^{\prime \prime} \text { specie. } G_{\mathrm{B}}^{\mathrm{III}} \text { : }
$$

$\{(123)(456)\} ; \quad r=1,2,3 ;(14)(26)(35) ;(25)(34)(16) ;(36)(15)(24)$.

غ̇ invariante il solo punto $D(123)$. Il gruppo è dispari.

È sottogruppo pari massimo $G_{3}^{\mathrm{I}}$. Il numero è 20 .

29. - Ordine uguale a 8. Esistono sei specie : esse provengono dall'associare due $G_{4}$ di specie diversa in tutti $\mathrm{i}$ sei modi possibili.

$I^{a}$ specie $G_{8}^{\mathrm{r}}$. Si associ il $G_{4}^{\mathrm{I}}$ del $n^{0}{ }^{0} 27 \mathrm{col} G_{4}^{\mathrm{II}}$ seguente :

$$
\text { (14) (23) (56); (14) (23); (56); I }
$$

e si avrà il cercato $G_{3}^{\mathbf{I}}$. Esistono i seguenti 5 punti invarianti :

$$
D(13,24), \quad D(12,34), \quad D(14,23), \quad D(56), \quad S(1234) \text {. }
$$


Il gruppo è dispari : il sottogruppo pari massimo è

(12) (34); (13) (24); (1 4) (23); I.

Il numero è 15 .

$I I^{\prime \prime}$ specie $G_{8}^{\mathrm{II}}$. Si associ il $G_{4}^{\mathrm{I}}$ solito del $\mathrm{n}^{0} 27 \mathrm{col}{G_{4}^{\mathrm{III}}}^{\mathrm{Ir}}$ seguente:

$$
(14)(23) ;(14)(56) ;(23)(56) ; \quad I \text {. }
$$

I punti invarianti sono i tre seguenti:

$$
S(1234) ; \quad D(14,23) ; \quad D(56) .
$$

Il $G_{8}^{\mathrm{nI}}$ è dispari. 亡̀ sottogruppo massimo il $G_{4}^{\mathrm{nI}}$ precedente. Il numero dei $G_{8}^{\mathrm{II}} \grave{\mathrm{è}} 4 \overline{5}$.

$I I I^{\prime \prime}$ specie $Q_{8}^{\mathrm{III}}$. Il medesimo $G_{4}^{\mathrm{I}}$ del n., 27 associato col $G_{4}^{\mathrm{IV}}$ seguente: $\left\{(1243)(56) \| r, r=1,2,3,4\right.$, dà luogo al $G_{: 3}^{\mathrm{III}}$ ora cercato. Sono invarianti tutti i punti della retta che unisce $D(145)$ con $D(146)$ e inoltre $D(56)$. II $G_{8}^{\text {III }}$ è dispari. Il sottogruppo pari massimo è il $G_{4}^{\mathrm{IV}}$ precedente. Il numero dei $G_{s}^{\text {III }}$ è 45 .

$I V^{\prime \prime}$ specie $G_{8}^{\mathrm{IV}}$. Esso proviene dall'associare i due $G_{4}^{\mathrm{Ir}}$ e $G_{4}^{\mathrm{II}}$ del $n .^{\circ} 27$. Sono invarianti tutti i punti della retta $d(12,34,56)$ e inoltre: $S(1234)$, $S(1256), S(3456)$. Il $G_{8}^{\mathrm{rv}}$. è dispari. E sottogruppo pari massimo $G_{4}^{\mathrm{nII}}$. Il numero dei $G_{\mathrm{s}}^{\mathrm{IV}}$ è 15 .

$V^{\prime \prime}$ specie $G_{8}^{\mathrm{V}}$. Si associ il $G_{4}^{\mathrm{II}}$ del $n^{0} 27$ col seguente $G_{4}^{\mathrm{YV}}$ :

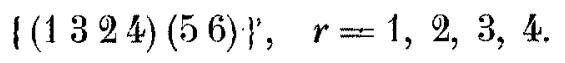

Sono invarianti i 5 punti seguenti: $S(1234), D(12,24), D(56)$ e i punti d'incontro di $d(12,34)$ con $F$. Il $G_{8}^{\mathrm{v}}$ è dispari. È sottogruppo pari massimo il $G_{4}^{\mathrm{IV}}$ precedente. Il numero è 45 .

$V I^{\prime \prime}$ specie $G_{8}^{\mathrm{VI}}$. Finalmente associando il $G_{*}^{\mathrm{rv}}$ del $n^{\circ} 27 \mathrm{col}$ seguente $G_{4}^{\text {III }}:\{(13)(24) ;(13)(56) ;(24)(56) ; I\}$ si otterrà il richiesto $G_{8}^{\mathrm{VI}}$. Sono invarianti i tre punti

$$
S(1234) ; \quad D(13,24) ; \quad D(56) .
$$

Il $G_{8}^{\mathbf{v I}}$ è pari. Il numero è 4 ó.

30. - Ordine uguale a 9. Esiste una sola specie $G_{9}^{\mathrm{I}}$. Un tal gruppo è il prodotto di due $G_{s}$. Basta ad esempio moltiplicare $(123)^{*}$ per $(456)^{*}$, $r=1,2,3$ in tutti i modi possibili. Sono punti invarianti $D(123)$ e i 4 punti d'intersezione di $S(123), S(456)$ con $F$. Il $G_{9}^{1}$ è pari. Il numero è 10 . 
31. - Ordine uguale a 12. Esistono due specie.

$I^{*}$ specie $G_{12}^{\mathrm{I}}$. Per ottenere questo gruppo basta aggiungere al $G_{6}^{\mathrm{I}}$ seguente (n. $\left.{ }^{\circ} 28\right):(123456)^{\prime}(r=1,2,3,4,5,6)$ la collineazione (1 2) (45) (36) i di cui simboli si ottengono scegliendo due lati opposti dell'esagono 123456 e i due vertici rimanenti. Esiste un sol punto invariante che è $D(135)$. Il $G_{13}^{\mathrm{I}}$ è dispari. È sottogruppo pari massimo un $G_{6}^{\mathrm{HI}}$. Il numero è 60 .

$I I^{n}$ specie. $G_{12}^{\mathrm{II}}$ (considerato da Cole, ommesso da CAYlery). Per generare un tal gruppo si assuma il seguente $G_{4}^{\text {III }}$ :

$$
(14)(25) ; \quad(25)(36) ; \quad(36)(14) ; 1
$$

e vi si aggiunga la collineazione $(123)(456)$ che lo trasforma in se stesso e si troverà il cercato $G_{12}^{\mathrm{II}}$. Sono invarianti i punti d'incontro di $d(14,25,36)$ con la $F$. Il $G_{12}^{\mathrm{II}}$ è pari. Il numero è 15.

32. - Ordine uguale a 16 . Si ha una sola specie $G_{15}^{1}$.

Esso può ottenersi aggiungendo al $G_{8}^{\mathrm{vI}}$, del n." 29 , la collineazione (56). Sono invarianti i medesimi punti che sono tali rispetto al $G_{8}^{\mathrm{vI}}$ suddetto e cioè

$$
S(1234) ; D(13,24) ; D(56) \text {. }
$$

Il $G_{16}^{\mathbf{I}}$ è dispari. 亡̀ sottogruppo pari massimo $G_{8}^{\mathrm{YI}}$.

Il numero dei $G_{1 \mathrm{~s}}^{\mathrm{I}}$ è 45 .

33. - Ordine uguale a 18. Si hanno tre specie.

$I^{n}$ specie : $G_{18}^{\mathrm{I}}$. Esso può riguardarsi come il prodotto del gruppo totale sopra 1, 2, 3 per $(456)^{\prime} r=1,2,3$. II $G_{18}^{r}$ è dispari, essendo sottogruppo pari massimo il $G_{9}^{\mathrm{I}}$ del n. ${ }^{\circ} 30$. T'enendo conto di questa circostanza si vede che i punti invarianti, rispetto all'attuale $G_{18}^{\mathbf{r}}$, sono i due punti d'incontro di $S(123)$ con la quadrica $F$ e il punto $D(123)$.

II numero dei $G_{18}^{\mathrm{I}}$ è 20.

$I I^{\prime \prime}$ specie: $G_{18}^{\mathrm{II}}$. Per ottenerlo si faccia il prodotto del gruppo totale sopra 1,2,3 per il gruppo totale sopra 4, 5,6 e dopo si scelgano le sostituzioni pari. Si trova così il cercato $G_{18}^{\mathrm{II}}$ che riesce un gruppo pari. $\grave{E}$ sottogruppo invariante il $G_{9}^{\mathrm{I}}$ del $n .^{0} 30$. Onde si deduce che esiste un sol punto invariante rispetto a $G_{18}^{\mathrm{II}}$ ed è $D(123)$.

Il numero dei $G_{18}^{\mathrm{II}}$ è 10 .

$I I I^{\prime \prime}$ specie: $G_{18}^{\mathrm{III}}$. Si aggiunga al $G_{9}^{\mathrm{I}}$ del $n^{\circ}{ }^{\circ} 30$ una collineazione a periodo 6 che lo trasformi in sè stesso: ad es. $(142536)$ e si otterrà $G_{15}^{\text {III. }}$. 11 simbolo di tale collineazione è quello di un esagono che ha per vertici alternati 123,456 essendo $(123)^{r},(456)^{r}$ i gruppi che hanno generato il 
$G_{9}^{\text {s }}$ (cf. il n. ${ }^{\circ} 30$ ). Tali esagoni sono sei (cf. il n. ${ }^{\circ} 17$ ): ogni $G_{18}^{\text {III }}$ ne assorbe tre. Il $G_{16}^{\mathrm{III}}$ è dispari. È sottogruppo pari massimo il suddetto $G_{9}^{\mathrm{I}}$. Il numero dei $G_{18}^{\text {III }} 20$.

34. - Ordine uguale a 24. Esistono 5 specie.

$1^{\prime \prime}$ specie: $G_{24}^{\mathrm{I}}$. Esso proviene daJ gruppo tetraedrico sopra 1234 per l'aggiunta di $(56)$. E un sottogruppo il $a_{s}^{\text {I }}$ del $n .^{\circ} 29$ mediante il quale risulta che $G_{24}^{1}$ possiede i soli due punti invarianti $S(1234), D(56)$. Il gruppo attuale è dispari. È sottogruppo pari massimo il gruppo tetraedrico suddetto. Il numero di questi $G_{2:}^{\mathrm{I}}$ è 15 .

$I I^{a}$ specie: $G_{2 t}^{\mathrm{II}}$. Per ottenere questo gruppo si possono prendere tutte le sostituzioni dispari sopra 1234 e moltiplicarle per (56) e dopo aggiungere il gruppo tetraedrico sopra gli stessi elementi 1234 . Si perviene cosi a un gruppo pari che è il $G_{z=}^{\mathrm{II}}$ cercato. Esso possiede $G_{\beta}^{\mathrm{vI}}$ come sottogruppo (n. ${ }^{\circ} 29$ ) per cui si vede che i punti invarianti rispetto all'attuale $G_{21}^{\text {II }}$ sóno due soltanto e cioè : $S(1234)$ e $D(56)$. Il numero dei $G_{24}^{\mathrm{IS}}$ è 15 .

$I I I^{a}$ specie: $G_{24}^{\mathrm{HI}}$. Si aggiunga al $G_{12}^{\mathrm{H}}$ del n. ${ }^{\circ} 31$ la sostituzione $(1245)$ e si otterrà $G_{24}^{\text {HI }}$. Vengono così ad aggiungersi le sostituzioni simili (1346), (2356) con le loro inverse e in conseguenza (16) (25) (34) e altre 5 simili. Non esiste alcun punto invariante. Il gruppo attuale è dispari. E sottogruppo pari massimo il $G_{12}^{\text {n }}$ sopra nominato. Anche questi $G_{24}^{\text {III }}$ sono in numéro di 15.

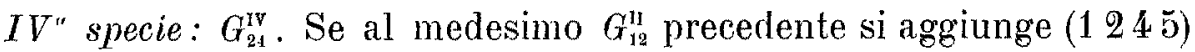
(36), in luogo di $(1245)$, si perviene al $G_{24}^{\mathrm{IV}}$ invece che a $G_{24}^{1 \mathrm{II}}$. Anche $G_{24}^{\mathrm{V}} \grave{\mathrm{e}}$ privo di punti invarianti (come $\left.G_{24}^{\mathrm{nI}}\right)$. Il gruppo attuale è pari. Il numero è 1 ó.

$V^{\prime \prime}$ specie: $G_{: t}^{\mathrm{v}}$ (considerato da Cole, ommesso da Cayley). Il solito $G_{12}^{\text {n }}$ ̀̀ invariante rispetto a (14). Aggiungendo una tale collineazione si perviene al cercato $G_{2 \pm}^{v}$. Si vengono così ad aggiungere (25) e 36) e altre 8 collineazioni a periodo 6 (a due a due inverse): i simboli di queste collineazioni a periodo 6 sono costituiti dagli esagoni che hanno per vertici opposti $14,25,36$. Sono punti invarianti quelli d'incontro di $d(14,25,36)$ con la quadrica $F$. Il $G_{24}^{\mathrm{v}}$ è dispari essendo sottogruppo pari massimo $G_{12}^{\mathrm{II}}$. Il numero è 15 .

35. - Ordine uguale a $36 . \mathrm{Vi}$ sono tre specie.

$I^{n}$ specie: $G_{36}^{\mathrm{I}}$. Essso è il prodotto del gruppo totale sopra 123 per il gruppo totale sopra 456 . E un gruppo dispari che ha per sottogruppo pari massimo il $G_{18}^{11}$ il cui punto invariante $D(123)$ è anche tale rispetto al presente $G_{36}^{\mathrm{I}}$. Il numero di questi $G_{36}^{1}$ è 10 .

$I I^{\prime \prime}$ specie: $G_{36}^{11}$. Per ottenerlo basta aggiungere al $G_{18}^{\mathrm{II}}$ la collineazione 
(2536) (14). Si vengono così ad aggiungere in conseguenza altre 8 collineazioni simili in guisa da averne 9: esse con le loro inverse completano il

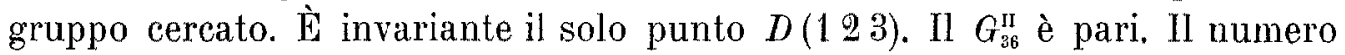
dei $G_{a c}^{\mathrm{II}}$ è 10.

III ${ }^{a}$ specie: $G_{3 j}^{\mathrm{III}}$ (considerato da Cole, ommesso da Cayley). Per ottenere un tal gruppo si osservi che $G_{18}^{\mathrm{II}}$ e $G_{18}^{\mathrm{m}}$ hanno in comune $G_{3}^{\mathrm{L}}$ e basta riunirli per ottenere il cercato $G_{36}^{\mathrm{LI}}$. Vengono, con ciò, ad aggiungersi 9 collineazioni esteme ai due $G_{18}^{\mathrm{n}}$ e $G_{18}^{\mathrm{nI}}$. I simboli di queste nuove collineazioni possono compendiarsi in quelle dei tre esagoni (152436), (162534), $(142635)$ i quali, insieme ai tre già esistenti in $G_{18}^{\prime \prime \prime}$, costituiscono i sei che hanno per vertici alternati 123,456 . 亡̀ punto invariante il solo $D(123)$. Il $G_{36}^{\prime \prime \prime}$ è dispari avendo $G_{18}^{\mathrm{u}}$ per sottogruppo pari massimo. Il numero dei $G_{36}^{\text {III }}$ è 10 .

36. - Ordine uguale a 48 . - Esistono due specie.

$I^{a}$ specie: $G_{18}^{\mathrm{I}}$. Esso proviene dal gruppo totale sopra 1, 2,3,4 per l'aggiunta della collineazione ( 56 ). Il gruppo è dispari essendo sottogruppo pari massimo $G_{24}^{\mathrm{II}}$. Sono punti invarianti $S(1234)$ e $D(56)$. II numero è 15 .

$I I^{a}$ specie : $G_{18}^{\mathrm{II}}$. Questo gruppo si può ottenere riunendo $G_{24}^{\mathrm{vv}}$ con $G_{24}^{\mathrm{v}}$ i quali hanno in comune $G_{1: t}^{\prime \prime}\left(n .^{\circ} 34\right)$. Le 12 nuove collineazioni che vengono ad aggiungersi sono dei due tipi seguenti : $\left(\begin{array}{ll}1245 & 4\end{array}\right)$ e $(12)(45)(36)$ : sei dell'uno e sei dell'altro. Il gruppo attuale è privo di punti invarianti. È dispari avendo per sottogruppo pari massimo il $G_{24}^{1 r}$. Il numero è 15 . Devesi anche notare che il $G_{48}^{\mathrm{nI}}$ è il gruppo imprimitivo di ordine massimo quando sieno riguardati come sistemi di imprimitività 14, 25,36. Possiede come retta invariante la diagonale di $1 .^{a}$ specie $d(14,25,36)$ e come piano invariante $\delta(14,25,36)$.

37. - Ordine uguale a 60. Esiste una sola specie: $G_{60}^{1}$. Per costruire un tal gruppo si assuma un $G_{10}$ sopra i cinque elementi 1, 2, 3, 4, 5. Ad es. : (cf, il n. ${ }^{0} 12$ della mia Memoria sul pentaedro)

$$
\begin{array}{cl}
(12345)^{r} ; \quad r=1,2,3,4,5 \\
(12)(35) ; & (23)(41) ; \quad(34)(52) ; \quad(45)(13) ; \quad \text { (51) (24). }
\end{array}
$$

Ebbene, ciascuna di queste collineazioni a periodo 2 individua un $G_{12}^{\mathrm{rI}}$ (cf. il $n .^{\circ} 31$ ) e così si ottengono i cinque sottogruppi tetraedrici del $G_{60}^{\mathrm{I}}$ domandato. Le collineazioni esterne a tali sottogruppi sono le seguenti (a periodo 5) insieme a tutte le loro potenze:

$$
\text { (12345), (13246), (13652), (14625), (16354), (26534). }
$$


Il $G_{60}^{\mathrm{I}}$ è privo di punti invarianti; è icosaedrico e quindi è pari. Il numero è 6 .

38. - Ordine uguale a 72. Vi è una sola specie: $G_{72}^{\mathrm{I}}$. Il gruppo può ottenersi riunendo $G_{36}^{\mathrm{II}}$ e $G_{36}^{\mathrm{m}}$ i quali hanno a comune il $G_{18}^{\mathrm{nI}}$. Le 18 collineazioni esterne ai due gruppi sopranominati sono delle due specie (12), (123) (45) e precisamente 6 della $1 .^{a}$ e 12 della 2. ${ }^{a}$ E invariante il solo punto $D(123)$. Il $G_{72}^{\mathrm{r}}$ è dispari essendo sottogruppo pari massimo $G_{36}^{\mathrm{Ir}}$. Il numero è 10. È da notarsi che $G_{72}^{I}$ è il gruppo d'ordine massimo quando si riguardino come sistemi di imprimitività : 123,456 .

39. - Ordine uguale a 120. Esiste una sola specie $G_{120}^{\mathrm{x}}$. Per costruire un tale gruppo basta aggiungere la collineazione (13) (25) (46) al $G_{60}^{\mathrm{y}}$ (n. ${ }^{\circ} 37$ ). $\mathrm{Si}$. perviene così al $G_{120}^{\mathrm{r}}$ che è dispari e ha per sottogruppo pari $G_{\mathrm{e} 0}^{\mathrm{r}}$. Il numero dei $G_{120}^{\mathrm{i}}$ è sei. Giascuno è privo di punti invarianti. Fra le collineazioni di $G_{120}^{\mathrm{I}}$ esterne a $G_{60}^{\mathrm{I}}$ sono da annoverarsi le seguenti 10 a periodo due :

$$
\begin{array}{ll}
(13)(25)(46) ; & (15)(23)(46) \\
(24)(31)(56) ; & (21)(34)(56) \\
(35)(42)(16) ; & (32)(45)(16) \\
(41)(53)(26) ; & (43)(51)(26) \\
(52)(14)(36) ; & (54)(12)(36) .
\end{array}
$$

Ora ricordiamo che tutte le collineazioni di questa specie del $G_{i 20}$ totale sono 15: ebbene i simboli delle cinque che avanzano sono identici a quelli delle seguenti cinque rette diagonali di $1 .^{a}$ specie:

$$
\begin{gathered}
d(16,34,25) ; \quad d(26,45,31) ; \quad d(36,51,42) \\
d(46,12,53) ; \quad d(56,23,14)
\end{gathered}
$$

ed è facile constatare che $G_{120}^{\mathrm{I}}$ funziona da gruppo totale sopra queste cinque rette come $G_{60}^{\mathrm{I}}$ funziona da gruppo alterno. Ne segue che due qualunque delle rette nominate non s'incontrano altrimenti tutte si incontrerebbero a due, a due e quindi dovrebbe esistere un punto, o un piano, invarianti rispetto a $G_{120}^{\mathrm{x}}$. Dunque $\mathrm{i}$ sistemi di cinque diagonali di $1 .^{\mathrm{a}}$ specie analoghi al precedente sono sei (questi sono i $G_{120}^{\mathrm{I}}$ ). Se per brevità si chiama quintupla un tal sistema di 5 rette diagonali sghembe a due, a due: si possono allora enunciare $i$ seguenti teoremi : 
"Con le 15 rette diagonali di 1." specie si possono formare sei quintuple. Sopra le diagonali di ciascuna quintupla funziona da gruppo totale un $G_{120}^{\mathrm{I}}$ e da gruppo alterno il relativo sottogruppo icosaedrico $G_{\mathrm{6} 0}^{\mathrm{I}}$. Ogni diagonale di 1." specie appartiene a due quintuple. Una quintupla è individuata da due delle sue rette."

Se si riflette che una terna di rette in posizione generica fra loro ammette nello spazio ambiente una ed una sola trasversale appoggiata alle tre rette della terna, si può anche aggiungere:

«Le 10 relte diagonali di 1." specie che avanzano quando si tolgono da tutte le 15, quelle di una quintupla, costituiscono le 10 trasversali delle 10 terne contenute nella quintupla medesima».

40. - Consideriamo i 15 punti diagonali di $1^{a}$ specie. Essi sono a tre, a tre allineati sopra le $\check{\partial}$ diagonali di una quintupla qualunque, (ad es.: la quintupla precedente). Formiamo i due pentagoni ordinati seguenti :

$$
D(12) D(45) D(23) D(15) D(34) ; \quad D(14) D(25) D(13) D(24) D(35) \text {. }
$$

I lati di questi pentagoni possono riferirsi in guisa che $\mathrm{i}$ corrispondenti s'incontrino nei $\check{5}$ punti $D(i k)$ rimanenti. Infatti si ha che

$$
\begin{aligned}
& \text { le due rette } \left.\begin{array}{l}
D(12) . D(45) \\
D(14) . D(25)
\end{array}\right\} \text { s'incontrano in } D(36) \\
& \text { » } \left.\quad \gg \quad \begin{array}{l}
D(45) \cdot D(23) \\
D(24) . D(35)
\end{array}\right\} \quad 》 \quad \gg D(16)
\end{aligned}
$$

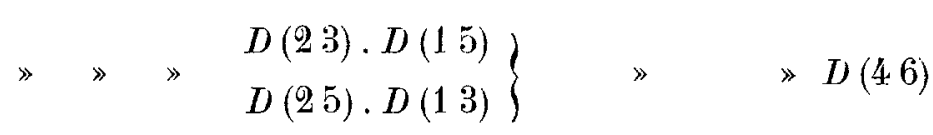

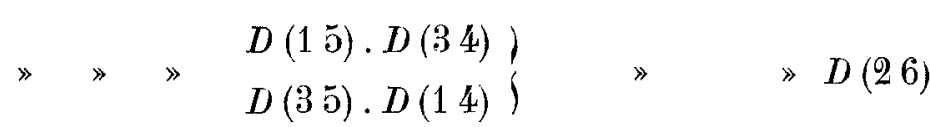

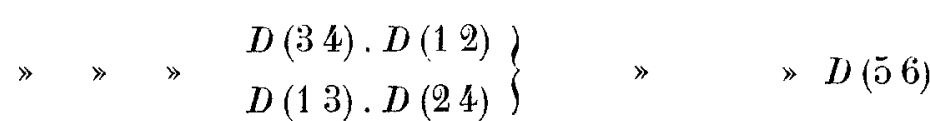

Si può dunque dire che: "con $i$ 15 punti diagonali di 1. " specie si possono formare tre pentagoni di cui 2 ordinati in guisa che $i$ lovo lati s'incontrino nei vertici del 3. pentagono (che resulla cosi, iscritto nei primi due). I vertici dei 3 pentagoni sono allineati a tre, a tre nelle cinque diagonali di una 
quintupla a cui la cfz. attuale rimane, in tal modo, coordinata. Esistono quindi 6 tali cfz.". ${ }^{n i}$.

41. - Ordine uguale a 360. Esiste una sola specie e un sol gruppo $G_{360}^{\mathrm{L}}$ che è il sottogruppo alterno. Esso contiene i $6 G_{60}^{\mathrm{r}}$ del $n .{ }^{\circ} 37$ e per generarlo basta aggiungere al $G_{60}^{\mathrm{s}}$ di quel numero una sostituzione pari esterna a tale $G_{60}^{\mathrm{I}}$.

\section{GAPITOLO VII.}

\section{Alcune considerazioni sopra le ipersuperficie invarianti dei primi cinque ordini.}

42. - Abbiamo già osservato al 11.0 2 che non esiste alcun iperpiano invariante rispetto a $G_{\text {т20 }}$, e che di quadriche invarianti ne esiste una sola rappresentata analiticamente da $\sum_{i}^{6} x_{i}^{o}=0$ e indicata col simbolo $\boldsymbol{H}^{*}$. Anche le ipersuperficie cubiche invarianti si riducono ad una sola (che è la $V_{s}^{s}$ di Segre). Infatti si consideri il sottogruppo $G_{120}$ che tiene fisso uno spazio fondamentale e permuta in tulti i modi possibili gli spazi rimanenti. Esso subordina in tale spazio un gruppo di collineazioni quaternarie dotato di una sola superficie cubica invariante (cf. la mia Memoria sul pentaedro n. ${ }^{\circ}$ 14) e quindi appartenente a tutte le ipersuperficie cubiche invarianti rispetto al $G_{720}$ totale. Se dunque queste ipersuperficie fossero due esse avrebbero in comune le sei superficie cubiche, analoghe a yuella descritta, nei sei spazi fondamentali dell'esaedro. Ciò è impossibile evidentemente. Dunque :

«L'unica ipersuperficie cubica invariante, è la $V_{3}^{3} d i$ SEgRe (la quale, come è ben noto ha 10 punti doppi nei 10 punti diagonali di 2." specie) ».

43. - Passiamo alle ipersuperficie quartiche. Esse potranno rappresentarsi tutte con una equazione della forma:

$$
a \sum_{1}^{6} x_{i}^{4}+b \sum_{1}^{6} x_{i}^{4} x_{h}+c \sum_{1}^{6} x_{i}^{2} x_{i}^{2}+d \sum_{1}^{6} x_{i}^{2} x_{h} x_{k}+e \sum_{1}^{6} x_{i} x_{h} x_{k} x_{l}=0,
$$

dove $a, b, c, d, e$ sono costanti. 
Se non che, a causa di $\sum_{1}^{6} x_{i}=0$, valgono le seguenti identità :

$$
\begin{gathered}
\sum_{1}^{5} x_{i}^{3} x_{h}=-\sum_{1}^{6} x_{i}^{4} ; \quad \sum_{1}^{6} x_{i}^{2} x_{h} x_{k}=\frac{1}{2} \sum_{1}^{6} x_{i}^{4}-\sum_{1}^{6} x_{i}^{2} x_{h}^{2} \\
\sum_{1}^{6} x_{i} x_{h} x_{h} x_{l}=-\frac{1}{8} \sum_{1}^{6} x_{i}^{4}+\frac{1}{4} \sum_{1}^{6} x_{i}^{2} x_{k}^{2} .
\end{gathered}
$$

Per cui il sistema cercato si riduce al seguente fascio:

$$
\alpha \sum_{i}^{6} x_{i}^{4}+\beta \sum x_{i}^{2} x_{k}^{2}=0
$$

al quale appartiene la quartica $F$ contata due volte $(\alpha=1, \beta=2)$.

Si ha dunque:

«Le ipersuperficie quartiche invarianti formano un fascio e tutte si toccano lungo una superficie di $8 .^{\circ}$ ordine situata sulla quadrica fondamentale».

44. - Fra le ipersuperficie precedenti merita speciale considerazione quella individuata da $\frac{\beta}{\alpha}=-\frac{2}{3}$ cioè la seguente :

$$
3 \sum_{1}^{6} x_{i}^{4}-2 \sum_{1}^{6} x_{i}^{2} x_{\bar{h}}^{2}=0
$$

e che noi indicheremo col simbolo $W$ secondo una notazione già introdotta da Castelnovo (*). Ci proponiamo adesso di esporre qui le proprietà più notevoli di questa ipersuperficie alcune delle quali rilevate già dall' autore suddetto.

Si cominci dall'osservare che $W$ passa per uno e quindi per tutti i punti diagonali di $1 .^{a}$ e di $3 .^{a}$ specie: da cui segue che essa contiene per intiero le 15 rette diagonali di $1 .^{\text {a }}$ specie perchè ciascuna possiede tre punti diagonali di $1 .^{a}$ e tre di $3 .^{a}$ specie $\left(n .^{\circ} 9\right)$. Dico che ciascuna di queste rette è doppia per $W$. Infatti sia $P$ un punto qualunque di $d(12,34,56)$. La collineazione (1 2) (34) (56) trasforma $W$ in se stessa: ogni retta $r$ passante per

(*) Loc. cit. L'equazione della ipersuperficie, nella forma data da Castejnovo, è :

$$
\left(\sum_{1}^{6} x_{i}^{2}\right)^{2}-16 \sum_{1}^{6} x_{i} x_{h} x_{k} x_{l}=0 .
$$

Essa si riduce subito alla nostra tenendo conto delle identità del $n .^{\circ} 43$. 
$P$ e che abbia un punto comune col piano $\delta(12,34,56)$ è unita per la collineazione suddetta $\left(\right.$ n. $\left.^{\circ} 19\right)$ e quindi il punto $P$ assorbe almeno due delle intersezioni di $r$ con $W$ : per conseguenza $r$ esiste nell' $S_{3}$ tangente a $W$ in $P$, da cui segue che tale $S_{3}$ (se è determinato) è quello individuato da $P$ e dal piano $\delta(12,34,56)$. Ma $d(12,34,56)$, appartenente a $W$, ̀̀ esterna allo spazio suddetto individuato da $P$ e da $\delta(12,34,56)$, perchè $d(19,34,56)$ e il suo piano polare $\delta(12,34,56)$ non hanno alcun punto comune. Ne segue che l' $S_{3}$ tangente in $P$ a $W$ è indeterminato e quindi $P$ è un punto doppio.

Si ha dunque il resultato:

"La ipersuperficie $W$ possiede come rette doppie le 15 diagonnli di 1." specie. Essa dunque è tangente, lungo una quadrica, a ciascuno spazio diagonale di 2." specie $\Delta(i h k)$. Tale quadrica è la $Q(i h k)$ del n." 12.

« Spettano dunque a tali 15 rette doppie tutte le proprietà dei $n$. 9-12: il fatto che esse sono distribuite a 6, a 6 nelle 10 quadriche suddette, induce a ritenere la ipersuperficie in discorso come la più naturale estensione (nello spazio a 4 dimensioni) della superficie quartica (dello spazio a 3) dotata di 15 punti doppi siluati a 6, a 6 sopra 10 coniche, in 10 piani singolari tangenti alla superficie lungo tali coniche».

$\grave{E}$ appunto di tale specie la superficie sezione di $W$ con uno qualunque dei 6 spazi fondamentali (cf. la mia Memoria sul pentacdro: n. ${ }^{\circ} 17$ ).

45. - Ogni piano diagonale di $3 .^{a}$ specie contiene sei punti diagonali di 3." specie esistenti su di una stessa conica $\left(n{ }^{\circ} 13\right)$, dunque si può dire che

"I 60 piani diagonali di 3." specie toccano ciascuno la ipersuperficie $W$ lungo una conica».

Siccome ogni retta di Pascal contiene 3 punti diagonali di 3 . $^{\text {a specie }}$ (n. $\left.{ }^{\circ} 14\right)$ che sono doppi per $W$ così ne viene che ciascuna di tahi rette appartiene per intiero a $W$. Tenendo presente il $n .^{\circ} 17$ ne segue che: «I quindici piani diagonali di 1. "specie tagliano ciascano le ipersuperficie $W$ secondo un quadrilatero che ha per lati $\dot{z}$ rette di PASCAL ».

Uno spazio diagonale di $10^{\mathrm{al}}$ specie come $\Delta(12)$ contiene tre piani diagonali di $1 .^{\mathrm{a}}$ specie : $\delta(12,34,56), \delta(12,35,46), \delta(12,36,45)$; contiene 4 piani diagonali di $3 .^{a}$ specie $\delta(3,12), \delta(4,12), \delta(5,12), \delta(6,12)$ e nou

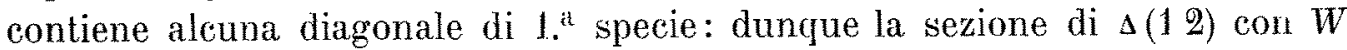
sarà una superficie quartica con 15 punti doppi situati a tre, a tre sopra 12 rette e a 6 , a 6 sopra 4 coniche. Con le 12 rette si potranno formare 3 quadrilateri piani aventi per vertici i 15 punti doppi suddetti, ecc., ecc. 
Finalmente la sezione con uno spazio fondamentale dell'esagono è una superficie di Sternen. Ad es. : la sezione con $\Sigma(3456)$ ha come rette doppie: $d(12,34,56), d(12,35,46), d(12,36,45)$ concorrenti in $D(12)$ che è il punto triplo della superficie sezione, ecc., ecc.

45. - Per cercare tutte le ipersuperficie quintiche invarianti, osserveremo (analogamente a ciò che fu fatto nel n. ${ }^{\circ} 43$ ) che esse potranno rappresentarsi con una equazione della forma:

$$
\begin{gathered}
a \sum_{1}^{6} x_{i}^{5}+b \sum_{1}^{6} x_{i}^{4} x_{h}+c \sum_{1}^{6} x_{i}^{3} x_{h}^{2}+d \sum_{1}^{6} x_{i}^{3} x_{h} x_{k}+ \\
+e \sum_{1}^{6} x_{i}^{2} x_{h}^{2} x_{k}+f \sum_{1}^{6} x_{i}^{2} x_{h} x_{k} x_{l}+g \sum_{1}^{6} x_{i} x_{h} x_{k} x_{l} x_{m}=0
\end{gathered}
$$

dove $a, b, c, d, e$ sono costanti.

Ma, a causa di $\sum_{1}^{6} x_{i}=0$, resulta identicamente:

$$
\begin{gathered}
\sum_{1}^{6} x_{i}^{4} x_{h}=-\sum_{1}^{6} x_{i}^{5} \\
\sum_{1}^{6} x_{i}^{3} x_{h}^{2}=\frac{1}{5} \sum_{1}^{6} x_{i}^{5}-6 \sum_{1}^{6} x_{i} x_{h} x_{k} x_{l} x_{m} \\
\sum_{1}^{6} x_{i}^{3} x_{h} x_{k}=\frac{2}{5} \sum_{i}^{6} x_{i}^{5}+3 \sum_{1}^{6} x_{i} x_{h} x_{k} x_{l} x_{m} \\
\sum_{1}^{6} x_{i}^{2} x_{h}^{2} x_{k}=-\frac{1}{5} \sum_{1}^{6} x_{i}^{5}+6 \sum_{1}^{6} x_{i} x_{h} x_{k} x_{l} x_{m} \\
\sum_{1}^{6} x_{i}^{2} x_{h} x_{k} x_{l}=-.5 \sum_{1}^{6} x_{i} x_{h} x_{k} x_{l} x_{m} .
\end{gathered}
$$

Per cui si perviene al fascio seguente:

$$
\alpha \sum_{1}^{6} x_{i}^{5}+\beta \sum_{1}^{6} x_{i} x_{h} x_{i k} x_{l} x_{m}=0
$$

Esso può essere definito dalla Hessiana di $V_{3}^{3}(\alpha=0)$ e dalla ipersuperficie costituita da $V_{3}^{\mathrm{s}}$ e da $F\left(\frac{\alpha}{\beta}=-\frac{1}{5}\right)$ la quale ipersuperficie è anche l'unica riduttibile nel fascio. Abbiamo dunque il resultato:

«Come le ipersuperficie quartiche invarianti, così, anche le quintiche, formano un fascio: esso è individuato dalla Hessiana della $V_{3}^{3}$ di SEgre e dalla 
ipersuperficie spezzata nella $V_{3}^{3}$ suddetta e nella quadrica invariante fondamentale».

La forma medesima della equazione della Hessiana dimostra il suo pas. saggio per tutte le facce $S(i k)$ dell'esaedro. Per lo spigolo $S(123)$ passano $S(12), S(13), S(23)$ che non esistono in un medesimo spazio: dunque $S(123)$ è doppio e i vertici dell'esaedro su di esso sono tripli per l'Hessiana in discorso, la quale ha anche per punti doppi i $10 \mathrm{D}(i h k)$. Quindi : "LiHessiana di $V_{\mathrm{s}}^{\mathrm{s}}$ ha 15 rette doppie nei 15 spigoli dell'esaedro e 20 punti tripli nei 20 vertici: ha inoltre 10 punti doppi nei 10 punti doppi di 2." specie (e non ha altri punti multipli).

Quinto al Mare, marzo 1909. 